\title{
Effect of intrathecal baclofen on the monosynaptic reflex in humans: evidence for a postsynaptic action
}

\author{
P Azouvi, A Roby-Brami, A Biraben, J B Thiebaut, C Thurel, B Bussel
}

Department of

Neurological

Rehabilitation

P Azouvi

A Biraben

B Bussel

INSERM U215,

Raymond Poincaré

Hospital, Garches,

France

A Roby-Brami

Department of

Neurosurgery, Henri

Dunant Hospital

J B Thiebaut

Department of

Neurosurgery,

Lariboisiere Hospital,

Paris, France

C Thurel

Correspondence to:

Dr B Bussel, Service de

Rééducation Neurologique

Hêpical Raymond Poincaré,

92380, Garches, France.

92380, Garches, France.

Accepted 1 April 1992.

\begin{abstract}
Intrathecal baclofen is a very powerful antispastic agent. Its mechanism of action on the monosynaptic H-reflex in spinal patients was investigated. It could inhibit rapidly and profoundly monosynaptic reflexes in lower limbs, but did not modify Ia vibratory inhibition of the soleus $\mathrm{H}$-reflex. To assess more precisely its effect on Ia afferents, an experimental paradigm using Ia heteronymous facilitation of the soleus H-reflex was used. Intrathecal baclofen did not modify the amount of monosynaptic facilitation of the soleus H-reflex brought about by stimulation of the femoral nerve. This demonstrates that the main part of the inhibitory effect of baclofen on the $H$-reflex in spinal patients is not due to a presynaptic effect, suggesting a postsynaptic site of action.
\end{abstract}

(F Neurol Neurosurg Psychiatry 1993;56:515-519)

Baclofen $(\beta$-(4-chlorophenyl)- $\gamma$-aminobutyric acid) is a widely used antispastic treatment. Recently, Penn et $a l^{1-3}$ have shown that its intrathecal administration is an effective treatment for severe spasticity. The mechanism of action of baclofen on monosynaptic reflex (MSR) remains unclear. Experimental studies

Table Patients' characteristics

Group I: $90 \%$ inhibition of soleus $\mathrm{H}$ reflex after an injection of baclofen $50 \mu \mathrm{g}$ or less

\begin{tabular}{lllll}
\hline No. & Age, sex & Diagnosis ${ }^{\star}$ & $\begin{array}{l}\mathrm{H}_{\text {max }} / \mathbf{M}_{\text {max }} \text { ratio } \\
\text { (initial value) }\end{array}$ & Delay of action (min) $\dagger$ \\
\hline 1 & $18 \mathrm{M}$ & SCI (T 5 c) & $0 \cdot 75$ & 60 \\
2 & $26 \mathrm{M}$ & SCI (T 4 c) & $0 \cdot 95$ & 60 \\
3 & $55 \mathrm{M}$ & SCI (T 6 c) & $0 \cdot 80$ & 240 \\
4 & $20 \mathrm{M}$ & SCI (C 4 i) & $0 \cdot 87$ & 35 \\
5 & $39 \mathrm{~F}$ & SCI (T 7 i) & $0 \cdot 75$ & 180 \\
6 & $29 \mathrm{~F}$ & SCI (C 3 i) & $0 \cdot 75$ & 80 \\
7 & $37 \mathrm{M}$ & MS & $0 \cdot 64$ & 60 \\
\hline
\end{tabular}

Group II: $90 \%$ inhibition of soleus $\mathrm{H}$ reflex after an injection of baclofen $60-100 \mu \mathrm{g}$

\begin{tabular}{lllll}
\hline 8 & $24 \mathrm{M}$ & SCI (C 7 c) & 0.59 & 40 \\
9 & $55 \mathrm{M}$ & SCI (T 6 i) & 0.75 & 69 \\
10 & $50 \mathrm{M}$ & SCI (T 7 i) & 0.61 & 80 \\
11 & $31 \mathrm{M}$ & SCI (C 5 i) & 0.76 & 60 \\
\hline
\end{tabular}

Group III: $90 \%$ inhibition of soleus $\mathrm{H}$ reflex after an injection of baclofen 100-150 $\mu \mathrm{g}$

\begin{tabular}{lllll}
\hline 12 & $36 \mathrm{~F}$ & SCI (C 5 c) & 0.4 & 120 \\
\hline
\end{tabular}

SCI (C 5 i) $\quad 0.95 \quad 120$

Group IV: patients in whom an injections of doses of baclofen 160-200 $\mu \mathrm{g}$ could not elicit more than $50 \%$ inhibition of soleus $\mathrm{H}$ reflex

$\begin{array}{llll}14 & 24 \mathrm{M} & \text { SCI (T } 4 \mathrm{c}) & 0.51 \\ 15 & 35 \mathrm{M} & \text { SCI (C } 5 \mathrm{i}) & 0.67\end{array}$

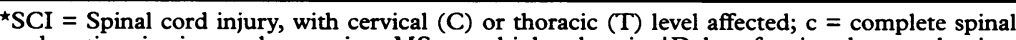
cord section, $\mathrm{i}=$ incomplete section. $\mathrm{MS}=$ multiple sclerosis. + Delay of action denotes the time (in minutes) necessary to obtain an inhibition of $90 \%$ of $\mathrm{H}$ reflex.

in animals are ambiguous. Some studies favour a postsynaptic action on neurons. ${ }^{4}$ More recent studies in spinal animals favour the hypothesis of a presynaptic effect on Ia afferents. ${ }^{56}$ Contrasting with these results, studies in spastic human patients, ${ }^{78}$ have failed to demonstrate a presynaptic effect. In these studies, presynaptic inhibition on Ia fibres was only assessed by vibratory inhibition of MSR.

Two facts led us to re-examine the mechanisms responsible for the inhibition of MSR by intrathecal baclofen in humans. Firstly, intrathecal baclofen allows a much stronger inhibition than oral administration, ${ }^{1-3}{ }_{9-12}$ thus allowing a more reliable study. Secondly, Hultborn $e t$ al $^{13}$ have recently described a new method which permits a more direct study of Ia presynaptic inhibition in humans.

\section{Patients and methods}

This investigation was performed in 15 patients, aged 18-55. This therapy was undertaken because all of them had severe spasticity and disabling spasms of spinal origin. The main characteristics of the patients' clinical state are summarised in the table. Fourteen had a traumatic spinal cord lesion, of which six were clinically complete, and eight incomplete. One had spastic paraparesis due to multiple sclerosis. The duration of the lesion was at least six months. All gave their consent after being informed of the experimental aim of the electrophysiological study. At the time of the experimental session, the patients were free from pressure sores, urinary tract infection or any other medical complication and had no oral treatment of spasticity. During the recording sessions, the patients sat in their wheelchairs or lay on a bed with their legs in the same position throughout the experimental session. A bolus of baclofen was injected into the lumbar subarachnoid space either by percutaneous lumbar puncture or by an implanted pump via a subcutaneous intrathecal catheter (manual pumps (Cordis Secor) in cases 8 and 11 , or electronically driven pumps (Medtronic DAT) in cases 12-15. The doses ranged from 20 to $200 \mu \mathrm{g}$ (table).

The H-reflex was tested on the soleus muscle. Surface electrodes were used both for stimulation and recording. Rectangular current pulses of $1 \mathrm{~ms}$ duration were used for stimulation, and were applied using a unipolar electrode. The active electrode was placed in the popliteal fossa to stimulate the tibial nerve. The reflex responses were measured as the peak-to-peak amplitude of the evoked muscle 
action potential. These potentials were recorded with two non-polarisable disc electrodes $(0.9 \mathrm{~mm}$ diameter; inter-electrode distance 2 $\mathrm{cm}$ ) placed over the bellies of the soleus muscle (below the gastrocnemius muscles). The electromyographical responses were measured using an analog digital converter linked to a microcomputer. The value of $\mathrm{Hmax}$ was assessed by $\mathrm{H}$-reflex recruitment curves obtained by 25 tibial posterior nerve stimulations with different intensities.

Presynaptic Ia inhibition was assessed by two methods: (1) Vibration (shocks percussion of amplitude $0.7 \mathrm{~mm}$, duration $2 \mathrm{~ms}$, frequency $70 \mathrm{~Hz}$ ) was applied to the Achilles tendon with a vibrator (Bruêl and Kjaer model 4809) driven by monophasic rectangular pulses. $H_{\max }$ with and without vibration, was recorded before, and every 15 minutes after, baclofen injection in eight patients (cases 1,2 , $3,5,6,8,9,11) .^{2}$ Heteronymous Ia facilitation of the soleus H-reflex was assessed according to the method described by Hultborn et al. ${ }^{13}$ The conditioning stimulus was obtained by supramaximal stimulation of femoral nerve in the femoral triangle.

It has been demonstrated that the first 0.5 $\mathrm{ms}$ of this heteronymous Ia facilitation from quadriceps to soleus is mediated exclusively through a monosynaptic pathway and is not contaminated by any Ib or polysynaptic Ia effect. ${ }^{13}$ Accordingly, the timing was adjusted in order to make the conditioning afferent volley arrive at the spinal level no more than $0.5 \mathrm{~ms}$ before the test soleus $\mathrm{H}$-reflex. The conditioning stimulus was delivered after the test stimulus, because the distance from the femoral triangle to the spinal cord is shorter than the distance from the popliteal fossa to the spinal cord. First, the shortest conditioning-test interval (using $0 \cdot 1 \mathrm{~ms}$ steps) at which facilitation of the soleus $H$-reflex could be elicited was determined. The interval used in the following part of the experiment was $0 \cdot 2-0.3 \mathrm{~ms}$ longer to obtain sizeable facilitation due only to the monosynaptic Ia EPSP.

The amount of facilitation before baclofen (control situation) was compared with that obtained every 10 minutes after the intrathecal injection. Under these conditions, the reflex facilitation depended only on the size of the conditioning EPSP evoked by heteronymous Ia stimulation. A constant conditioning stimulus should elicit an EPSP of constant size in motor neurons, and thus a constant amount of reflex facilitation, unless the amount of presynaptic inhibition of Ia fibres changes; a decrease in the facilitation may be ascribed to an increase in presynaptic inhibition of the heteronymous Ia fibres from quadriceps to soleus. It has been shown ${ }^{1415}$ that the susceptibility of an H-reflex to facilitation varies with the size of the unconditioned reflex. After baclofen injection, however, the soleus $\mathrm{H}$ reflex was depressed. To maintain a constant size $\left(10-15 \%\right.$ of $\left.M_{\text {max }}\right)$ of the reference (unconditioned) H-reflex despite MSR inhibition, the test stimulus intensity had to be adjusted (increased).

Every 10 minutes, a series of at least 20 unconditioned and 20 conditioned H-reflexes was recorded with stimuli being delivered every 4s. The amount of facilitation was then calculated, the statistical analysis of the results being examined by a $t$ test. Between these series, the $\mathrm{H}_{\text {max }} / \mathrm{M}_{\max }$ ratio was measured to assess the amount of MSR inhibition caused by baclofen. This experiment was performed in five patients (cases $8,10,11,13,14$ ) with doses ranging from 50 to $150 \mu \mathrm{g}$. In two other patients (cases 4 and 7 ), the experiment could not be performed because heteronymous facilitation was too small before baclofen injection. In the other patients, it was not possible because femoral nerve stimulation provoked strong flexor spasms or a long lasting soleus H-reflex inhibition, or both.

\section{Results}

\section{Effect on MSR}

The excitability of MSR in lower limbs was tested in all subjects. The soleus H-reflex was dramatically reduced by intrathecal baclofen. As the table illustrates, however, the minimal dose necessary to obtain this inhibition was different between one patient and another. In all patients except two, we observed (table) a near-total disappearance of the reflex $\left(\mathrm{H}_{\max }\right.$ less than $10 \%$ of the pre-treatment value) with doses of less than $150 \mu \mathrm{g}$. In seven patients this effect appeared with doses of less than $50 \mu \mathrm{g}$; in two cases higher doses (more than $150 \mu \mathrm{g}$ ) were necessary and, even with these doses, inhibition was not complete (reduction of the $\mathrm{H}_{\max } / \mathrm{M}_{\max }$ ratio of $50 \%$ ). The onset and the rate of the decrease of the $\mathrm{H}_{\max }$ value were also variable. As the table shows, in six patients, the effect appeared rapidly and, when the appropriate dose was given, the inhibition was complete within less than one hour. In four cases, more than two hours were necessary to obtain a complete inhibition of the H-reflex.

The minimal dose necessary to abolish the $\mathrm{H}$-reflex and the time to peak effect were not correlated with the $\mathrm{H}_{\max } / \mathrm{M}_{\max }$ ratio before injection, or with the level of the lesion, and did not differ whether the spinal lesion was clinically complete or not. This inter-individual variability may be due to patients' susceptibility to the drug but modifications of CSF flow may contribute to this variability, at least in paraplegia of traumatic origin.

For each patient there was a dose-effect relationship. This is illustrated in fig 1 by the $\mathrm{H}_{\max }$ variation observed after different doses of baclofen $(20,50$ and $100 \mu \mathrm{g})$ in one representative patient (case 10).

Effect on soleus $H$-reflex conditioned by Ia afferent stimulation

The effect of a vibratory stimulus was studied in eight subjects. Weak vibratory inhibition (less than $20 \%$ ) was observed in only two of them (cases 8 and 11). The injection of baclofen did not enhance vibratory inhibition in those two, nor did it make it appear in the other six patients (fig 2).

The effect on the soleus H-reflex Ia heteronymous facilitation was studied in five patients, the test H-reflex being deliberately set 


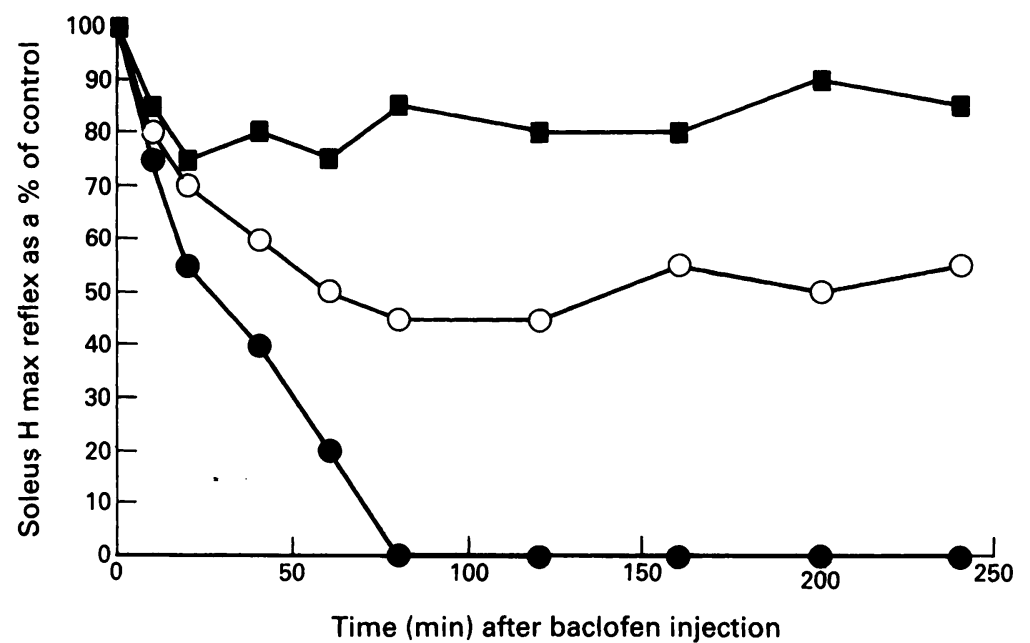

Figure 1 Dose-effect relationship, illustrated in patient 10. The H-reflex is expressed as a percentage of its pre-injection (control) value. The three curves represent the evolution of the $H_{\text {max }}$ value during the four hours following an intrathecal injection of baclofen $20 \mu \mathrm{g}$ (squares), $50 \mu \mathrm{g}$ (open circles), or $100 \mu \mathrm{g}$ (filled circles).

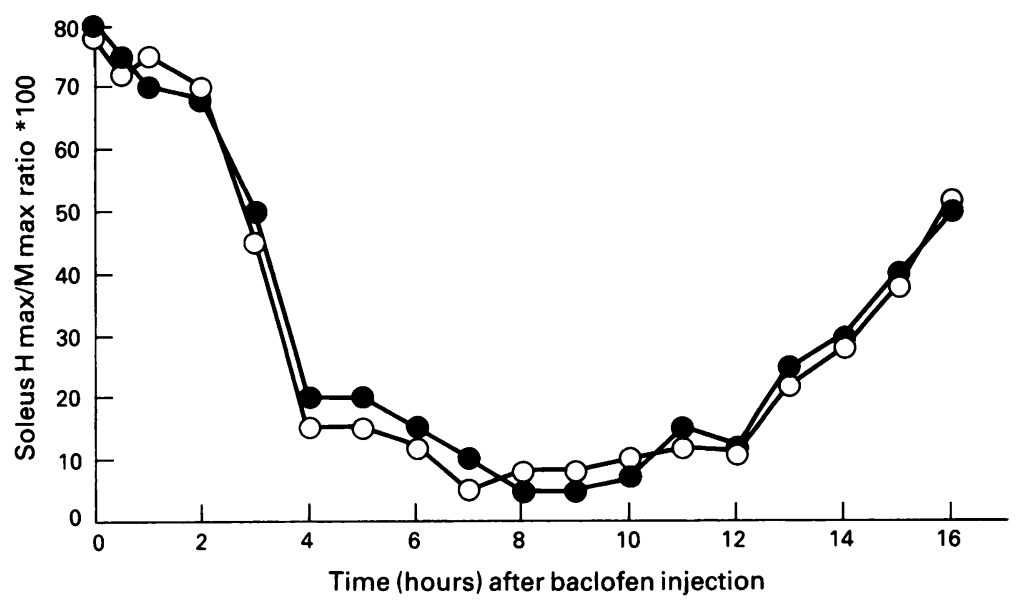

Figure 2 Effect of a vibratory stimulus on $H$-reflex after intrathecal baclofen. The figure represents soleus $H_{\max } / M_{\max }$ ratio with (filled circles) and without (open circles) vibrations during 16 hours following an intrathecal injection of baclofen $40 \mu \mathrm{g}$ in patient 3.

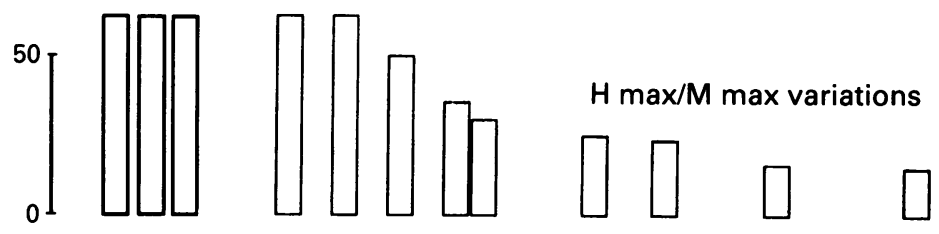

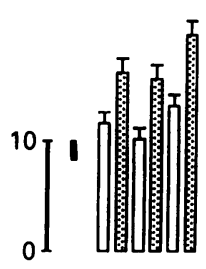

Controls

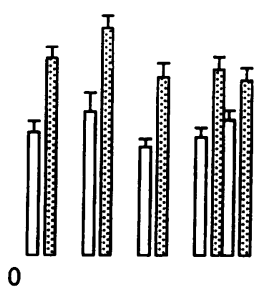

After baclofen injection
Test Test + la heteronymous
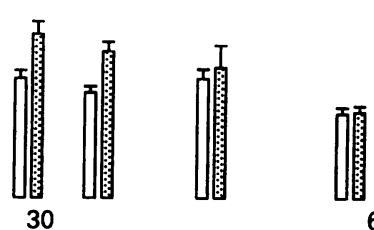

$60 \mathrm{mn}$

Figure 3 Heteronymous Ia facilitation of soleus $H$-reflex compared with $H_{\max } / M_{\max }$ ratio, before and after an intrathecal injection of baclofen $100 \mu \mathrm{g}$ in patient 8 . Top: $H_{\text {max }} / M_{\max }$ variation. Bottom: open bars represent test $H$-reflex, solid bars $H$-reflex conditioned by heteronymous Ia volley. The first three measures were obtained before, and the others during, the 60 minutes following baclofen injection. Abcissa: time (minutes) after baclofen injection; ordinate: size of $H_{\text {max }}$, expressed as a percent of $M_{\max }$. Note that different scales are used for the upper and lower parts of the figure. at $10-15 \%$ of $M_{\max }$ both before and after baclofen. In four patients (cases $8,11,13,14$ ), the same findings were observed: facilitation was not altered by the injection, as long as the $\mathrm{H}$-reflex was not profoundly inhibited $\left(\mathrm{H}_{\max }\right.$ ' $M_{\max }$ ratio higher than $25 \%$ ). Fig 3 represents in patient 8 the simultaneous evolution of the changes of the $\mathrm{H}_{\max } / \mathrm{M}_{\max }$ ratio (upper part) and the Ia heteronymous facilitation (lower part) after intrathecal injection of baclofen 100 $\mu \mathrm{g}$. The control soleus $\mathrm{H}_{\max }$ reflex was $62 \%$ of the $M_{\max }$ value, and it progressively decreased after injection. The test $\mathrm{H}$-reflex was increased $54 \%$ by the heteronymous Ia volley before injection and this amount of facilitation remained stable despite significant inhibition of the MSR: for an $\mathrm{H}_{\max } / \mathrm{M}_{\max }$ ratio of $25 \%$, the amount of facilitation was still $44 \%$. For stronger H-reflex inhibition, however, the facilitation weakened significantly and then disappeared. Figure 4 summarises the results in the five patients, showing the size of test and conditioned soleus $\mathrm{H}$-reflexes before and after an injection producing a reduction of the $\mathrm{H}_{\mathrm{max}}$ ' $M_{\max }$ ratio to $25 \%$. In four patients, the amplitude of the facilitation was not significantly modified by an injection of baclofen. In one patient (case 10), the facilitation decreased after injection. It should be noted, however, that the variability of the test $\mathrm{H}$-reflex was greater in this patient than in the other four. In this same patient, the injection was made by a percutaneous lumbar puncture, whereas an implantable pump was used in the other four. These technical problems may contribute to the variable results obtained in this case.

\section{Discussion}

Baclofen is known to be efficient in reducing muscle tone and flexor or extensor spasms in patients suffering from corticospinal tract lesions, as well as reducing stretch reflexes in animal experiments. ${ }^{416}$ Because this effect can be observed after spinal cord section, it is generally accepted that most antispastic activity is exerted at the spinal level. The precise site and mechanisms of action of this drug, however, still remain unclear. The present results document a powerful inhibitory effect of baclofen administered intrathecally, with rapid (from 35 minutes to four hours), profound and prolonged inhibition of MSR in the legs of spinal patients. Such a drastic effect has never been observed with oral treatment, and confirms the clinical observations made by Penn $e t$ $\mathrm{al}^{1-3}$ and the electrophysiological study of Macdonell et al ${ }^{11}$

Before baclofen injection, $\mathrm{H}$-reflex vibratory inhibition was weak or absent in our patients, who all presented a severe spasticity. This is in accordance with the findings of Delwaide ${ }^{17}$ who showed that vibratory inhibition was reduced in spastic patients, and that this loss of inhibition was related to the severity of hyperreflexia. Intrathecal baclofen did not enhance or restore Ia vibratory inhibition of MSR. The same results have already been obtained after oral $^{718}$ or intramuscular ${ }^{8}$ administration of 
A

Patient 8<smiles>C1CCC1</smiles><smiles>C1#CCC1</smiles>
$164 \%$

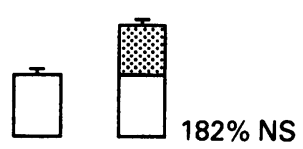

Patient 11
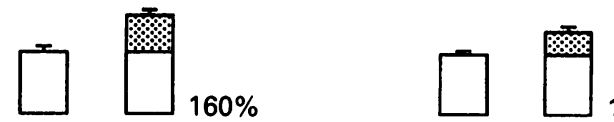

$137 \%$ NS
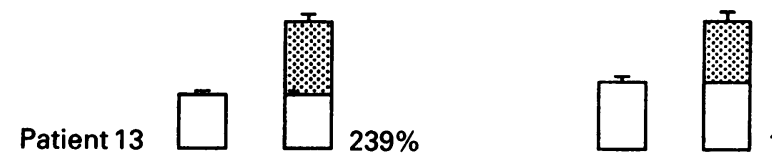

$193 \%$ NS
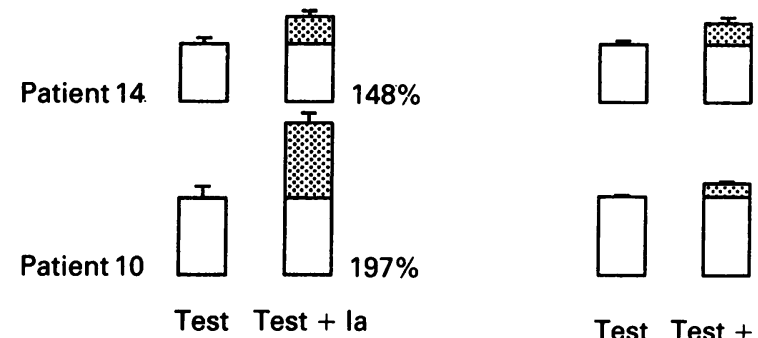

$135 \%$ NS

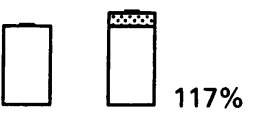

Test Test + la

Figure 4 Heteronymous Ia facilitation of soleus $H$-reflex in five patients before and after baclofen injection. Open bars: test reflex value. Solid bars: amount of facilitation brought about by heteronymous Ia volley. Each bar represents the mean of 20 recordings, plus one SD. A: before injection. B: after intrathecal baclofen injection, reducing $H_{\max } / M_{\max }$ ratio to $25 \%$. The average of facilitation (after 20 tests) is indicated for each case. For the four upper cases, the difference between facilitation before and after injection was not significant (NS).

baclofen but, in these studies, inhibition of MSR was much less important. As it has been shown that vibratory inhibition is essentially due to Ia presynaptic inhibition brought about by Ia fibres, ${ }^{17}$ this suggests that baclofen does not enhance this inhibitory mechanism.

Presynaptic inhibition on Ia fibres can be brought about by other pathways than Ia fibres themselves. To assess more precisely the effect of baclofen on Ia afferent terminals, we used the experimental procedure described by Hultborn et al. ${ }^{13}$ This technique has the advantage of allowing a study of presynaptic inhibition on Ia fibres, whatever its origin.

In our study, in four out of five patients in whom a reproducible Ia heteronymous facilitation of soleus motor neurons was obtained, this facilitation persisted unchanged, as long as the $\mathrm{H}_{\text {max }} / \mathrm{M}_{\text {matio }}$ was greater than $25 \%$. This result shows that $\mathrm{H}$-reflex inhibition is not due to a presynaptic mechanism, suggesting an effect at a postsynaptic level. When the $\mathrm{H}$ reflex was more deeply inhibited, facilitation decreased and disappeared, but this was not necessarily caused by a presynaptic effect of baclofen: Hultborn et $a l^{13}$ have shown that, with strong postsynaptic inhibition (reducing the amplitude of the test reflex by more than $50 \%$ of its control value), the peak of the Ia EPSP (and of the heteronymous Ia facilitation) is reduced. Hence, no conclusion can be drawn from the results obtained when the test reflex was deeply inhibited. This method tests only the presynaptic inhibition on heteronymous Ia afferents. It is likely that presynaptic inhibition on homonymous Ia afferents from soleus behaves in a similar way as Meunier and

Pierrot-Deseilligny ${ }^{19}$ have suggested that presynaptic inhibition on these two types of Ia fibres directed to soleus $a$-motor neurons could be mediated by the same interneurons.

In animal experiments and studies in vitro, there is no agreement on the presynaptic or postsynaptic effect of baclofen. ${ }^{4}$ Initial studies $^{20}$ and, more recently, Edwards $e t a l^{5}$ and Jimenez et $a l^{\circ}$, conclude that baclofen's main site of action is presynaptic, by reducing the probability of release of neurotransmitter, without any effect on the membrane time constant or EPSP decay. Nevertheless, postsynaptic effects have also been demonstrated by many authors who showed that baclofen has a hyperpolarising action, dependent on dose and concentration on some mammalian central neurons, associated with an increase in postsynaptic potassium conductance. ${ }^{4}$

Most data have been obtained in experimental conditions that are quite remote from human clinical conditions: firstly, these studies have only been performed on acute spinal animals, and secondly, intrathecal administration has not been studied in animal preparations.

In summary, our results suggest that intrathecal baclofen in chronic spinal patients inhibits the soleus $\mathrm{H}$-reflex by a postsynaptic effect, at least until the $\mathrm{H}_{\max } / \mathrm{M}_{\max }$ ratio is higher than $25 \%$. This effect may be exerted directly on the motor neuron itself, or by a postsynaptic inhibitory mechanism that is released by baclofen.

We are most grateful to Professor D Burke and Professor E Pierrot-Deseilligny for their helpful comments and critical review of the manuscript. We thank Professor O Lyon-Caen who referred his patients for this study, and Dr A Yakovleff for improving the English.

improving the English. This work has been supported in part by a grant from the
Fondation pour la Recherche Médicale, attributed to P Fondation
Azouvi.

This work has been presented in part at the second meeting of the European Neurological Society, Brighton, July 1990.

1 Penn RD, Kroin JS. Intrathecal Baclofen alleviates spinal cord spasticity. Lancet 1984;1:1078.

2 Penn RD, Kroin JS. Continuous intrathecal baclofen for severe spasticity. Lancet 1985;2:125-7.

3 Penn RD, Savoy SM, Corcos D et al. Intrathecal baclofen for severe spinal spasticity. $N$ Engl $\mathcal{f}$ Med 1989;320: 1517-21.

4 Zieglgansberger W, Howe JR, Sutor B. The neuropharmacology of baclofen. In Müller $\mathrm{H}$, Zierski J and Penn RD, eds. Local spinal therapy of spasticity. Berlin: SpringerVeriag, 1988:7-50.

5 Edwards FR, Harrison PJ, Jack JJB, Kullmann. Reduction by baclofen of monosynaptic EPSPs in lumbosacral motoneurones of the anaesthetized cat. $\mathcal{f}$ Physiol motoneurones of

6 Jimenez I, Rudomin P, Enriquez M. Differential effects of $(-)$ baclofen on Ia and descending monosynaptic EPSPs. Exp Brain Res 1991;85:103-13.

7 Ashby P, White DG. "Presynaptic" inhibition in spasticity and the effect of $\beta$ (4-chlorophenyl) GABA. $\mathcal{f}$ Neurol $S c i$ 1973;20:329-38.

8 Delwaide PJ. Electrophysiological analysis of the mode of action of muscle relaxants in spasticity. Ann Neurol 1985;17:90-5.

9 Müller H, Zierski J, Dralle D, Hoffmann O, Michaelis G. Intrathecal baclofen in spasticity. In: Müller $\mathrm{H}$, Zierski J, Penn RD eds. Local spinal therapy of spasticity. Berlin: Springer-Verlag, 1988:155-214.

10 Ochs G, Struppler A, Meyerson BA, et al. Intrathecal baclofen for long-term treatment of spasticity: a multicentre study. $\mathcal{f}$ Neurol Neurosurg Psychiatry 1989; 52:933-9.

11 Macdonell RAL, Talalla A, Swash M, Grundy D. Intrathecal baclofen and the $\mathrm{H}$ reflex. $\mathcal{F}$ Neurol Neurosurg Psychiatry 1989;52:1110-2.

12 Azouvi PH, Biraben A, Roby-Brami A, Bussel B, Thiebaut 

JB, Thurel C. Intrathecal administration of baclofen by an
implantable pump for treatment of severe spasticity. $\mathcal{F}$ Neurol 1988;235 (suppl 1):S75.

13 Hultborn H, Meunier S, Morin C, Pierrot-Deseilligny E. Assessing changes in presynaptic inhibition of Ia fibres: a study in man and the cat. $\mathcal{F}$ Physiol 1987;389:729-56.

14 Meinck HM. Facilitation and inhibition of the human H-reflex as a function of the amplitude of the control reflex. Electroencephalogr Clin Neurophysiol 1980;48: 203-11

15 Mazieres L, Morin C, Pierrot-Deseilligny E. Effet de l'amplitude du reflexe test sur le niveau de facilitation ou d'inhibition des réponses monosynaptiques. $\mathcal{f}$ Physiol (Paris) 1984;79:59A

16 Young RR, Delwaide PJ. Drug therapy: spasticity. $N$ Engl $\mathcal{F}$
Med 1981;304:28-33, 96-9.

17 Delwaide PJ. Human monosynaptic reflexes and presynaptic inhibition. An interpretation of spastic hyperreflexia. In: Desmedt JE, ed. New developments in electromyography and clinical neurophysiology, vol. 3. Basel: Karger, 1973:508-22.

18 Castaigne $\mathrm{P}$, Held JP, Pierrot-Deseilligny E, Bussel B Macquart-Moulin J. Etude de l'effet du Lioresal dans la spasticité. Rev Neurol 1973;128:245-50.

19 Meunier S, Pierrot-Deseilliony E Gating of the afferen volley of the monosynaptic stretch reflex during movement in man. $\mathcal{F}$ Physiol 1989;419:753-63.

20 Pierau FK, Zimmermann P. Action of a GABA-derivative on post-synaptic potentials and membrane properties of on post-synaptic potentials and membrane properties of
cat's spinal motoneurones. Brain Res 1973;54:376-80. 